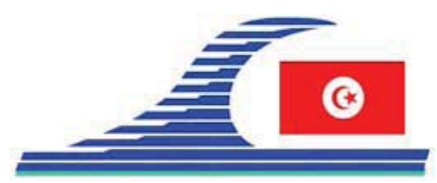

\author{
Conférence Méditerranéenne Côtière et Maritime \\ EDITION 1, HAMMAMET, TUNISIE (2009) \\ Coastal and Maritime Mediterranean Conference \\ Disponible en ligne - http://www.paralia.fr-Available online
}

\title{
Caractérisation du milieu marin de Monaco et identification des contraintes environnementales : mesures in situ et modélisation
}

\author{
Eric DAVID ${ }^{1}$, Pierre-François DEMENET ${ }^{1}$, Florence GANDILHON ${ }^{1}$, \\ Philippe GOURBESVILLE ${ }^{2}$, Bernard THOMASSIN ${ }^{3}$
}

1. SOGREAH, 6 rue de Lorraine, 38130, Echirolles, France.

eric.david@sogreah.fr

2. Université de Nice Sophia-Antipolis \& Polytech'Nice-Sophia, UMR 6012, 930 Route des Colles, 06903 Sophia Antipolis, France.

gourbesv@unice.fr

3. Centre Océanologie de Marseille, Université de la Méditerranée,

Station marine d'Endoume, rue de la batterie des Lions, 13007 Marseille. France.

ba.thomassin@wanadoo.fr

\section{Résumé :}

Dans le cadre d'une volonté affirmée de gestion de son littoral, la Principauté de Monaco a mis en œuvre une démarche holistique et durable de la connaissance de son milieu marin sur la base de conseils d'experts, de campagnes de mesures in situ et d'outils de modélisation dont l'ensemble présente un niveau rarement atteint aujourd'hui dans ce domaine. Nous présentons dans cet article la synthèse des connaissances à fin 2006 et les outils opérationnels mis en œuvre. L'objet est de pouvoir ainsi quantifier et maîtriser à long terme les conséquences d'un projet d'aménagement côtier ou marin. Cette démarche continue à ce jour avec pour objectif de maitriser aussi les conséquences des phases travaux sur l'environnement.

\section{Mots-clés :}

Ecosystèmes côtiers - Environnement marin - Hydraulique maritime - Modélisation Sédiments - Travaux maritimes

\section{Introduction}

Une étape essentielle pour l'évaluation environnementale d'un aménagement dans le milieu marin est l'établissement d'un diagnostic initial du milieu. Ce diagnostic doit s'attacher à caractériser le milieu et son évolution. La première étape passe par une compilation des connaissances. Dans le cas de Monaco, cet état des connaissances a été réalisé par THOMASSIN et GOURBESVILLE (2007). Ce document regroupe l'ensemble des connaissances à fin 2006 sur les domaines océanographique, météorologique, hydrologique, nature des fonds marins, qualité de l'eau et des sédiments, et un inventaire du peuplement faunistique et algal, benthique. 
Sur les 84 espèces d'invertébrés et de poissons reconnues comme " espèces d'intérêt patrimonial » par la France (arrêté du 26/11/1992) et la Convention de Berne (ratifiée par Monaco), 29 espèces ont été recensées sur le littoral de Monaco (de seulement $3,4 \mathrm{~km}$ de long). Ceci prouve la richesse faunistique des différents fonds de ce plateau continental. Dans le cadre d'une volonté affirmée de gestion de son littoral dans une démarche holistique et durable, la Principauté de Monaco a décidé en 2006 de mettre œuvre les moyens nécessaires à une meilleure connaissance de son milieu marin, structurant sa réflexion à partir d'un panel d'experts internationaux référents pluridisciplinaires et du déploiement d'un outil haute-performance de reproduction des écoulements hydrodynamiques tridimensionnels sur l'ensemble de son territoire.

\section{Le milieu marin}

Les principales conclusions de la synthèse sont les suivantes.

\subsection{Conditions météorologiques et courantologiques}

Les vents sur le site de Monaco sont globalement associés aux flux d'Est et d'Ouest issus du Mistral. Néanmoins, les effets orographiques sont dominants. En période estivale, le régime des vents est dominé par les brises thermiques. L'établissement d'un diagnostic pertinent - échelles spatiales et temporelles - sur la courantologie a requis la réalisation de deux campagnes de mesure - 8 ADCP disposés par les fonds couvrant les profondeurs 30 à $75 \mathrm{~m}$, été et l'hiver 2006. En synthèse, les courants côtiers observés ne sont jamais nuls mais restent faibles avec une intensité de l'ordre de 2 à $10 \mathrm{~cm} / \mathrm{s}$. La cohérence spatiale des écoulements montre la présence d'une circulation littorale erratique mais nette, probablement issue de la circulation macroscopique dans le bassin méditerranéen avec, en particulier, le courant Liguro-Provençal (voir notamment ALBEROLA et al., 1995, DAVID et al., 1995).

\subsection{Qualité de l'eau}

La qualité des eaux du littoral monégasque à l'instar de celle des côtes Nord ouest de la Méditerranée connait un processus de dégradation marqué depuis plusieurs décennies. La transparence des eaux du littoral et au large de Monaco a diminué depuis la fin 1998 et, en particulier, depuis 2001 comme le démontre le suivi réalisé par la Direction de l'Environnement de la Principauté. La profondeur limite de la zone euphotique est ainsi remontée d'environ $15 \mathrm{~m}$ durant ces 8 dernières années du fait d'une diminution de la transparence des eaux en relation avec leur charge particulaire.

\subsection{Nature et qualité des fonds}

Selon la bathymétrie et le degré d'envasement, une succession de fonds sédimentaires plutôt sableux se développe jusqu'à $25 \mathrm{~m}$ de profondeur. Au-delà et sur le plateau continental, des fonds vaseux occupent la majorité des surfaces (Détritique Envasé). Ces 
vases montrent des teneurs élevées en carbone organique ainsi qu'en azote Kjeldahl. Les teneurs en polluants divers dans les sédiments n'ont été analysées que dans des sédiments des ports Hercule et de Fontvieille et dans leurs abords immédiats. Selon la nomenclature des métaux lourds, ces sédiments sont classés de niveau 1, à l'exception du mercure (niveau 2) au niveau de la prise d'eau du Musée océanographique.

\subsection{Peuplements benthiques}

Les fonds vaso-sableux littoraux $(5-25 \mathrm{~m})$ sont encore colonisés par des herbiers de phanérogames marines. De par leur importance écologique, la « Réserve sous-marine du Larvotto » a été créée dès 1975 (50 ha), classée Aire Marine Protégée. Sur les fonds rocheux littoraux, les peuplements sont à dominance de macrophytes et d'invertébrés. La biocénose Coralligène, auparavant très développée sur le tombant des Spélugues, a permis de le classer en 1986 «Réserve corail». Depuis quelques années, on constate une régression de ce peuplement par envasement des pentes et présence de déblais pouvant être en relation avec la construction de la contre-jetée du port.

\section{Outil de connaissance du milieu marin - Modèle courantologique}

L'outil se base sur un modèle hydrodynamique tridimensionnel incluant les effets de température et de salinité, et de renouvellement des eaux.

L'analyse des mesures issues des campagnes courantométriques de 2006 (voir §2.1) permet de mettre en exergue l'existence de deux schémas courantologiques dominants, dénommés « scénarios hydrométéorologiques » en été et en hiver. Ce sont :

- des structures courantologiques oscillantes temporellement dont la direction est nettement orientée vers 1'Ouest (plus de 50\% du temps) d'une durée de 2 à 3 jours ;

- des structures courantologiques oscillantes temporellement dont la direction est nettement orientée vers l'Est (environ 25\% du temps) d'une durée d'1 à 2 jours.

Le reste du temps est composé de périodes transitoires principalement entre ces deux états. Le modèle hydrodynamique, élaboré sur TELEMAC-3D (propriété d'EDF) s'appuie sur une combinaison de plusieurs modèles établis à trois échelles :

- les données issues du modèle de la plateforme MERCATOR fournissent les données courantologiques journalières à l'échelle du bassin Ligure ;

- un modèle régional réalisé aussi sous TELEMAC-3D couvre la frange littorale Vintimille-Nice ;

- enfin le modèle local incluant les eaux territoriales monégasques et le Cap Martin.

Le calage du modèle, réalisé sur la base des données de 2006, est de très bon niveau au regard des conditions extrêmement complexes observées in situ et qui font intervenir un nombre très important de processus physiques (figure 1). Les qualités principales du modèle sont la bonne reproduction des intensités des courants et de leurs variations et des directions moyennes observées. Le modèle permet d'évaluer le degré de sensibilité 
du milieu marin par l'analyse des temps de résidence des eaux dans différents secteurs sensibles - réserves marines par exemple ou de manière globale.

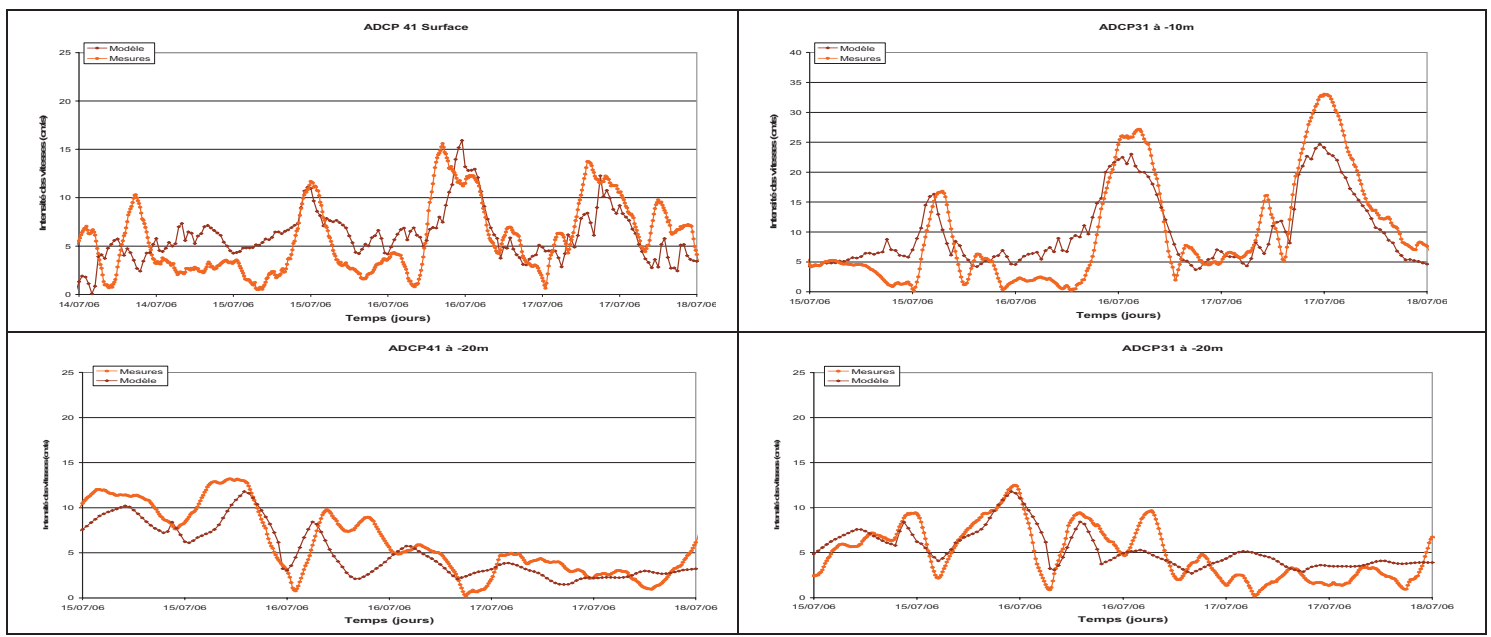

Figure 1. Comparaison mesure/modèle pour 2 ADCP-vitesses à la surface et à $-20 \mathrm{~m}$.

\section{Conclusion et perspectives}

Dans le cadre d'une volonté affirmée de gestion de son littoral, ceci dans une démarche holistique et durable, la Principauté de Monaco a su mettre en œuvre les moyens nécessaires à une meilleure connaissance de son milieu marin et se doter d'outils capables d'analyser et de quantifier les conséquences d'un projet d'aménagement. Le niveau atteint aujourd'hui représente un effort rarement atteint dans ce domaine.

Ces outils lui permettent d'envisager une maitrise de l'impact environnemental de projets marins. Afin d'améliorer ses connaissances et la qualité de l'expertise, la Principauté a décidé de poursuivre en 2009 les investigations in situ afin de couvrir notamment les aspects sédimentaires pour la gestion des impacts des phases travaux. Cette campagne de mesures permettra d'élaborer une chaîne de modèles hydrosédimentaires d'aide à la décision de premier plan et probablement inégalée en Europe.

\section{Références bibliographiques}

ALBEROLA C., MiLlOT C., FONT J. (1995). On the seasonal and mesoscale variabilities on the Northern Current during the PRIMO-0 experiment in the western Mediterranean Sea. Oceanologica acta , vol. 18, n² 2, pp 163-192.

DAVID E., RAPAIRE J., SAUVAGET P. (1995). Numerical Modelling of Hydrodynamics in Deep Coastal Waters of the Mediterranean - An application to the Bay of Monaco. Proceeding of the XXVIth IAHR Congress, London, U.K., 11-15 Sept. THOMASSIN B., GOURBESVILLE P. (2007). Etat des connaissances du milieu marin du litoral de la principauté de Monaco en 2006. Rapport pour la Direction de la Prospective de l'Environnement et de l'Urbanisme, Février 2007. 